\title{
Complex mucinous cystadenoma of undetermined malignant potential of the urachus
}

\author{
Andrew J. Schell, MD FRCPC; ${ }^{*}$ Curtis J. Nickel, MD, FRCSC; ${ }^{\dagger}$ Phillip A. Isotalo, MD FRCPC ${ }^{*}$
}

\begin{abstract}
Urachal mucinous neoplasms are rare and include both villous adenomas and invasive adenocarcinomas. These mucinous tumours should be completely excised as they can demonstrate aggressive clinical behaviour, including the development of pseudomyxoma peritonei. We describe a 70-year-old woman who presented with a lower abdominal mass and received a diagnosis of a rare, complex urachal mucinous cystadenoma of undetermined malignant potential. This tumour demonstrated extensive mucin extravasation into perivesical soft tissue, but was not associated with pseudomyxoma peritonei or any malignancy. Rarely described in the English literature, mucinous cystadenomas of the urachus should be treated similarly to their villous adenoma counterparts: with complete surgical excision to prevent local tumour recurrences.
\end{abstract}

Can Urol Assoc J 2009;3(4):E39-E41

\section{Introduction}

The urachus, an embryological structure extending from the umbilicus to the urinary bladder apex and containing the allantois, persists uncommonly in adults. ${ }^{1}$ Rarely the urachus may be the primary site of benign and malignant neoplasms. Urachal adenocarcinomas account for $20 \%-39 \%$ of primary urinary bladder adenocarcinomas, which in turn account for $0.17 \%-0.34 \%$ of all bladder malignancies. ${ }^{2}$ Even rarer than urachal adenocarcinomas are urachal villous adenomas that may coexist with in situ and invasive adenocarcinoma. ${ }^{3,4}$ Regardless of tumour type, all urachal mucinous tumours have the potential to behave in an aggressive clinical manner that includes the development of pseudomyxoma peritonei. ${ }^{5,6}$ We describe a 70-year-old woman who presented with a rare, complex urachal mucinous cystadenoma that demonstrated extensive mucin extravasation into perivesical soft tissue. The differential diagnosis of a urachal mucinous cystadenoma of undetermined malignant potential includes villous adenoma; urachal adenocarcinoma; urothelial malignancy with an adenocarcinoma component; and metastatic disease from a variety of sites, including the breasts, ovaries, gastrointestinal tract and prostate gland.

\section{Case report}

A 70-year-old woman presented to her family physician after noticing a new lower abdominal mass. She had a family history of abdominal aortic aneurysm. The patient had no irritative urinary symptoms and specifically had no gross hematuria or mucusuria. She had no recent weight loss. The patient had a history of peptic ulcer disease and no other clinically important medical history. A computed tomography (CT) scan of her abdomen and pelvis (Fig. 1) revealed a complex cystic mass extending from the anterosuperior dome of the urinary bladder to the umbilicus. A fibrous band joined the mass to the umbilicus and scattered curvilinear calcifications were present at the margin of the mass. The patient underwent resection of this cystic mass with partial cystectomy. At surgery, there was no evidence of pseudomyxoma peritonei or ovarian malignancy. In addition, the patient had no clinical evidence of a breast or gastrointestinal malignancy. A year after the resection, the patient was well, with no evidence of local recurrence or distant metastases.

\section{Pathological findings}

Gross examination revealed a $15.5 \times 8 \times 8$-cm multiloculated cystic mass within perivesical soft tissue that did not directly involve the urinary bladder wall (Fig. 2). This

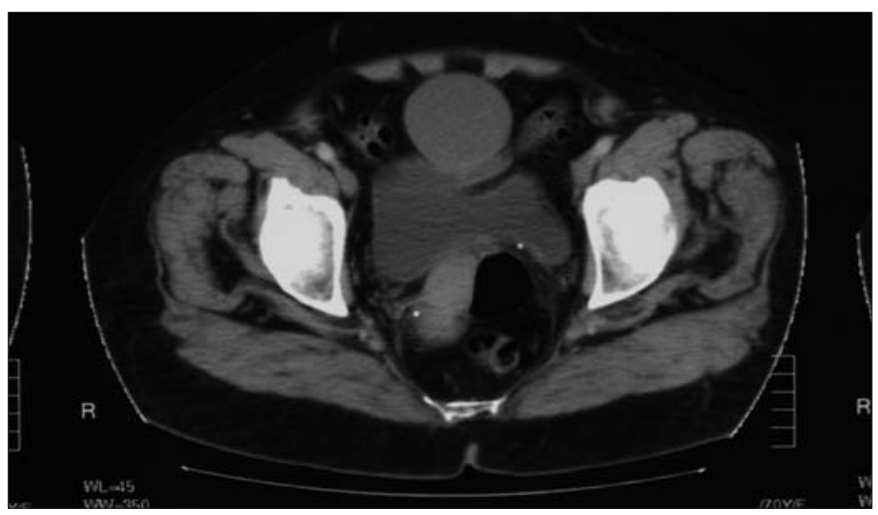

Fig. 1. Computed tomography scan of the pelvis of a 70-year-old woman demonstrating an irregular cystic mass within the region of the urachus. 
multiloculated cystic mass was composed of smooth-walled cysts that lacked papillary proliferations and that contained abundant mucin. Histological examination revealed a complex mucinous cystadenoma that was characterized by multiloculated, mucin-filled cysts that were lined by glandular epithelium that lacked pseudostratified and villous areas (Fig. 3). We identified no foci of dysplasia or adenocarcinoma in situ after extensive sampling and histological examination of this mucinous cystadenoma. The mucin that filled the cystadenoma was hypocellular and did not contain freefloating aggregates of atypical epithelial cells, the latter being a feature of mucinous adenocarcinomas. This mucinous cystadenoma did not demonstrate destructive stromal invasion, although mucin extravasation into perivesical soft tissue was present and was associated with foci of dystrophic calcification and a foreign body giant cell reaction. Based on the size and the extensive mucin extravasation into perivesical soft tissue, we diagnosed this mucinous tumour as a mucinous cystadenoma of undetermined malignant potential. This cystic neoplasm did not communicate directly with the urinary bladder, but was associated with an extravesical urachal remnant that confirmed the primary urachal origin of this complex mucinous cystadenoma. We completely excised the mucinous neoplasm. The uninvolved urinary bladder demonstrated no evidence of cystitis glandularis, intestinal metaplasia or urothelial malignancy.

\section{Discussion}

The differential diagnosis of a urachal mass is extensive and includes both neoplastic and nonneoplastic conditions. Rarely, persistence of a patent urachus will account for a supravesical mass. Abscesses secondary to bacterial, mycobacterial and parasitic infections may affect the urachus, and calculi may develop in cystic urachal remnants. ${ }^{1}$ Benign

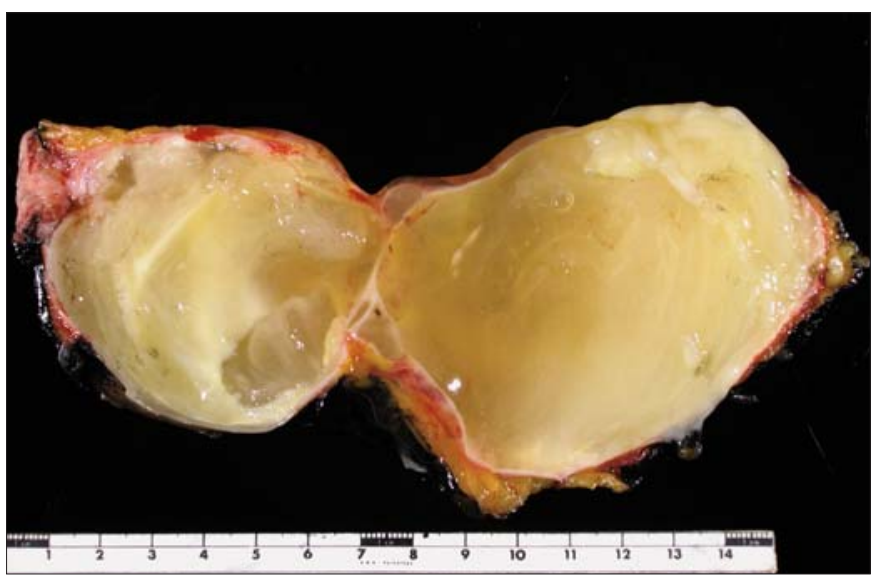

Fig. 2. Excised complex mucinous cystadenoma containing abundant mucin. neoplasms of the urachus include desmoid tumours, fibromas, leiomyomas and villous adenomas, although the latter may be associated with foci of dysplasia, adenocarcinoma in situ or frankly invasive adenocarcinoma. ${ }^{1,3,4,7-10} \mathrm{~A}$ rare urachal inflammatory myofibroblastic tumour has also been recently described in a 10-year-old child..$^{10}$ Most primary malignant urachal neoplasms are adenocarcinomas, although squamous cell carcinomas and sarcomas have also been described involving the urachus. ${ }^{1,2}$ Urachal adenocarcinomas comprise a significant subset of primary urinary bladder adenocarcinomas, accounting for $20 \%-39 \%$ of all primary bladder adenocarcinomas. ${ }^{2}$ Histological confirmation of a urachal remnant, tumour growth deep to the urinary bladder mucosa, tumour location with the dome of the bladder, absence of bladder intestinal metaplasia and the absence of another primary malignancy as a source for metastasis are all features helpful in identifying an adenocarcinoma as a tumour of primary urachal origin. ${ }^{11,12}$ As metastatic disease from breast, ovarian, prostate and colonic primaries can mimic urachal mucinous tumours, these sites of potential disease should be excluded clinically. ${ }^{3}$

We described a rare complex mucinous cystadenoma of undetermined malignant potential of the urachus that lacked the architectural features of a villous adenoma and lacked any evidence of destructive stromal invasion to suggest an invasive neoplastic process. To our knowledge, ours is only the second reported case of urachal cystadenoma in the English literature. ${ }^{13}$ This urachal cystadenoma of undetermined malignant potential, although associated with mucin

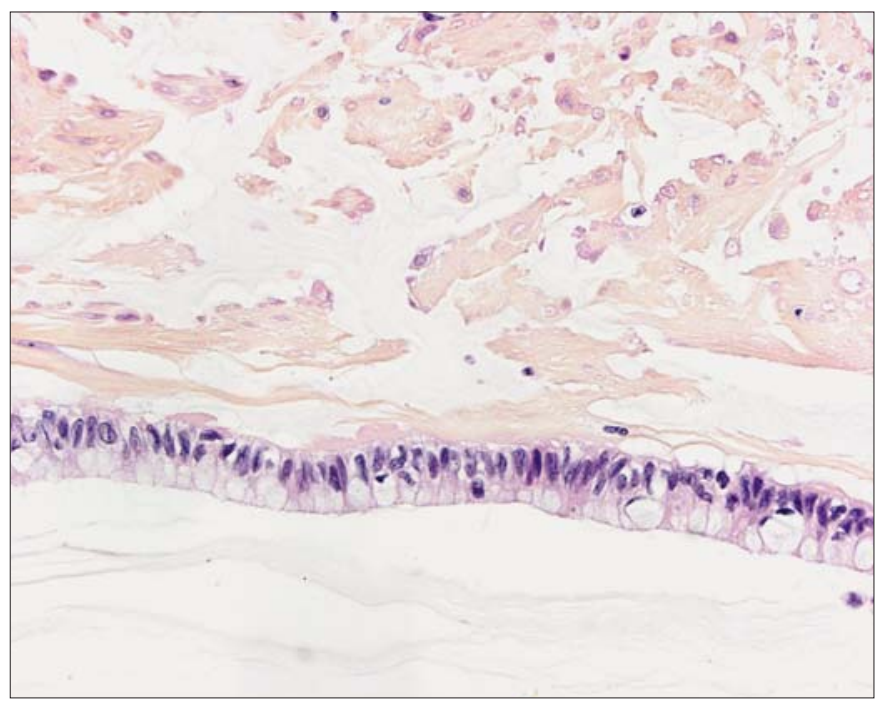

Fig. 3. Photomicrograph of the wall of the complex mucinous cystadenoma demonstrating glandular epithelium that is associated with mucin production and extensive mucin extravasation within the underlying stroma (original magnification $\times 200$ ). 
extravasation into perivesical soft tissue, was not associated with pseudomyxoma peritonei. Despite the absence of pseudomyxoma peritonei in our patient, all urachal mucinous tumours have the potential for aggressive clinical behaviour, including local tumour recurrence, and complete surgical excision of these tumours is of paramount importance. Considering the potential for local tumour recurrence and pseudomyxoma peritonei, the term mucinous tumour of uncertain malignant potential has been suggested for urachal mucinous tumours that lack frank invasion. ${ }^{5,6,14}$ As urachal mucinous neoplasms may be heterogeneous in their architecture, it is critical that these tumours are extensively sampled histologically to exclude foci of dysplasia or invasive malignancy. ${ }^{3}$ Mucinous tumours of the urothelial tract may be difficult to specifically classify on superficial transurethral biopsies that contain limited diagnostic material. ${ }^{3}$

Even in the absence of stromal invasion, the possibility of metastatic disease should be considered (e.g., metastatic breast, ovarian, gastrointestinal and prostate malignancies) and clinically excluded for all patients with mucinous tumours involving the urachus and urinary bladder, including mucinous cystadenomas of undetermined malignant potential. As mucinous tumours of the urothelial tract, including those of the urachus, may demonstrate aggressive clinical behaviour, patients should be followed clinically postexcision for evidence of local disease recurrence. Although rare, urachal mucinous cystadenomas of undetermined malignant potential should be included in the differential diagnosis of a urinary bladder dome mass, especially if the mass demonstrates a cystic component.

From the *Department of Pathology and Molecular Medicine and the ${ }^{\dagger}$ Department of Urology, Kingston General Hospital and Queen's University, Kingston, Ont.
This article has been peer reviewed.

Competing interests: None declared.

\section{References}

1. Eble JN. Abnormalities of the urachus, In: Young RH, editor. Pathology of the urinary bladder. New York. Churchill Livingstone; 1989. p. 213-43.

2. Sheldon CA, Clayman RV, Gonzalez R, et al. Malignant urachal lesions. J Urol 1984;131:1-8.

3. Cheng L, Montironi R, Bostwick DG. Villous adenoma of the urinary tract: a report of 23 cases, including 8 with coexistent adenocarcinoma. Am J Surg Pathol 1999;23:764-71.

4. Seibel JL, Prasad S, Weiss RE, et al. Villous adenoma of the urinary tract: a lesion frequently associated with malignancy. Hum Pathol 2002;33:236-41.

5. de Bree E, Witkamp A, Van De Vijver M, et al. Unusual origins of pseudomyxoma peritonei. J Surg Oncol 2000;75:270-4.

6. Mendeloff J, McSwain NE Jr. Pseudomyxoma peritonei due to mucinous adenocarcinoma of the urachus. South Med J 1971;64:497-8.

7. Stenhouse G, McRae D, Pollock AM. Urachal adenocarcinoma in situ with pseudomyxoma peritonei: a case report. J Clin Pathol 2003;56:152-3.

8. Paul $A B$, Hunt CR, Harney JM, et al. Stage 0 mucinous adenocarcinoma in situ of the urachus. J Clin Pathol 1998;51:483-4.

9. Eble JN, Hull MT, Rowland RG, et al. Villous adenoma of the urachus with mucusuria: a light and electron microscopic study. J Urol 1986;135:1240-4.

10. Nascimento AF, Dal Cin P, Cilento BG, et al. Urachal inflammatory myofibroblastic tumor with ALK gene rearrangement: a study of urachal remnants. Urology 2004;64:140-4.

11. Jones WA, Gibbons RP, Correa RJ Jr, et al. Primary adenocarcinoma of bladder. Urology 1980;15:119-22.

12. Abenoza P, Manivel C, Fraley EE. Primary adenocarcinoma of urinary bladder: clinicopathologic study of 16 cases. Urology 1987;29:9-14.

13. Hull MT, Warfel KA. Urachal cystadenoma with abundant glycogen: ultrastructural study. Ultrastruct Pathol 1994;18:499-502.

14. Carr NJ, Mclean AD. A mucinous tumour of the urachus: adenoma or low grade mucinous cystic tumour of uncertain malignant potential? Adv Clin Path 2001;5:93-7.

Correspondence: Dr. Phillip Isotalo, Department of Pathology and Molecular Medicine, Kingston General Hospital, 76 Stuart St., Kingston ON K7L 2V7; fax 613 548-6076; isotalop@kgh.kari.net 